\title{
UNIQUENESS OF MEROMORPHIC FUNCTIONS THAT SHARE ONE SMALL FUNCTION WITH THEIR DERIVATIVES
}

\author{
LiPEI LIU AND YongXING GU
}

\begin{abstract}
In this paper, we study uniqueness of meromorphic functions that share one small function $\mathrm{CM}$ with their derivatives. We mainly obtain a uniqueness theorem which answers the questions provided by Kit-wing $\mathrm{Yu}$.
\end{abstract}

\section{Introduction and main results}

In this paper, a meromorphic function always means a function, which is meromorphic in the whole complex plane. Let $f(z)$ and $g(z)$ be non-constant meromorphic functions, $a \in \bar{C}$. We say that $f(z)$ and $g(z)$ share the value a CM if $f(z)-a$ and $g(z)-a$ have the same zeros with the same multiplicities. When $a=\infty$, this means that $1 / f(z)$ and $1 / g(z)$ share the value $0 \mathrm{CM}$. We shall use the standard notations of value distribution theory, $T(r, f), m(r, f)$, $N(r, f), \bar{N}(r, f)$ (see L. Yang [1] or C. C. Yang and H. X. Yi [2]). We denote by $S(r, f)$ any function satisfying:

$$
S(r, f)=o\{T(r, f)\}
$$

as $r \rightarrow+\infty$, possibly outside a set (of $r$ ) of finite measure.

R. Brück [3] proved the following result:

THEOREM A. Let $f$ be a nonconstant entire function satisfying $\rho_{1}(f)<\infty$, where $\rho_{1}(f)\left(\rho_{1}(f)=\lim \sup _{r \rightarrow \infty}(\log \log T(r, f) / \log r)\right)$ is not a positive integer. If $f$ and $f^{\prime}$ share the value $0 C M$, then $f^{\prime} \equiv c f$ for some constant $c \neq 0$.

Gary G. Gundersen and Lian-Zhong Yang proved the following result:

THEOREM B [4]. Let $f$ be a nonconstant entire function of finite order. If $f$ and $f^{\prime}$ share one finite value a $C M$ then:

1991 MR Subject Classification: 30D35.

Key words: Entire Function; Meromorphic function; Share value; Unicity.

Received December 17, 2003; revised May 27, 2004. 


$$
\frac{f^{\prime}-a}{f-a}=c
$$

for some non-zero constant $c$.

It is natural to consider whether there exit any similar results for entire functions of infinite order, or even meromorphic functions $f$ and a small function $a$ of $f$.

In [5], Kit-wing $\mathrm{Yu}$ answered this question by proving the following two results:

THEOREM C. Let $k \geq 1$ and let $f$ be a non-constant entire function, $a(z)$ be a meromorphic function with $a(z) \not \equiv 0, \infty$, and $T(r, a)=o(T(r, f))$ as $r \rightarrow+\infty$. If $f-a$ and $f^{(k)}-a$ share the value $0 C M$ and $\delta(0, f)>3 / 4$, then $f \equiv f^{(k)}$.

THEOREM D. Let $k \geq 1$ and let $f$ be a non-constant meromorphic function, $a(z)$ be a meromorphic function with $a(z) \not \equiv 0, \infty, f$ and $a(z)$ do not have any common pole and $T(r, a)=o(T(r, f))$ as $r \rightarrow+\infty$. If $f-a$ and $f^{(k)}-a$ share the value $0 C M$ and $4 \delta(0, f)+2(8+k) \Theta(\infty, f)>19+2 k$, then $f \equiv f^{(k)}$.

In the same paper, the author posed the following questions:

Question 1: Is the condition $\delta(0, f)>3 / 4$ sharp in the Theorem C.

Question 2: Is the condition $4 \delta(0, f)+2(8+k) \Theta(\infty, f)>19+2 k$, sharp in the Theorem D.

Question 3: Can the condition " $f$ and $a(z)$ do not have any common pole" be deleted in the Theorem D?

In this paper, we apply the different method and obtain the following result, which answers above questions.

THEOREM 1. Let $k \geq 1$ and let $f$ be a non-constant meromorphic function, $a(z)$ be a meromorphic function with $a(z) \not \equiv 0, \infty$, and $T(r, a)=S(r, f)$ as $r \rightarrow+\infty$. If $f-a(z)$ and $f^{(k)}-a(z)$ share the value $0 C M$ and $f^{(k)}$ and $a(z)$ do not have any common poles of same multiplicity and

$$
2 \delta(0, f)+4 \Theta(\infty, f)>5
$$

then $f \equiv f^{(k)}$.

As a simple corollary, we have following result

COROLlary 1. Let $k \geq 1$ and let $f$ be a non-constant entire function, $a(z)$ be a meromorphic function with $a(z) \not \equiv 0, \infty$, and $T(r, a)=S(r, f)$ as $r \rightarrow+\infty$. If $f-a(z)$ and $f^{(k)}-a(z)$ share the value $0 C M$ and $\delta(0, f)>1 / 2$, then $f \equiv f^{(k)}$. 


\section{Some lemmas}

In this section, we have the following lemmas which will be needed in the proofs of the main results. In the following, $I$ is a set of infinite linear measure and may not be the same each time it occurs.

Lemma 1 [2]. Let $f_{1}$ and $f_{2}$ be two non-constant meromorphic functions and let $c_{1}, c_{2}, c_{3}$ be non-zero constant. If $c_{1} f_{1}+c_{2} f_{2}=c_{3}$ holds, then

$$
T\left(r, f_{1}\right) \leq \bar{N}\left(r, \frac{1}{f_{1}}\right)+\bar{N}\left(r, \frac{1}{f_{2}}\right)+\bar{N}\left(r, f_{1}\right)+S\left(r, f_{1}\right) .
$$

Lemma 2 [2]. Let $f_{i}(i=1 \cdots n)$ be $n$ linearly independent meromorphic functions. If they satisfy:

then for $1 \leq j \leq n$ we have

$$
\sum_{i=1}^{n} f_{i} \equiv 1
$$

$$
T\left(r, f_{j}\right) \leq \sum_{i=1}^{n} N\left(r, \frac{1}{f_{i}}\right)+N\left(r, f_{j}\right)+N(r, D)-\sum_{i=1}^{n} N\left(r, f_{i}\right)-N\left(r, \frac{1}{D}\right)+S(r),
$$

where $D$ is the Wronskian determinant $W\left(f_{1}, f_{2}, \ldots f_{n}\right), \quad S(r)=o(T(r))$ as $r \rightarrow+\infty, r \in I$, and $T(r)=\max _{1 \leq j \leq n}\left\{T\left(r, f_{j}\right)\right\}$.

Lemma 3. Let $f$ be a meromorphic function in the complex plane. For any positive integer $k$ such that $f^{(k)} \not \equiv 0$, we have:

$$
N\left(r, \frac{1}{f^{(k)}}\right) \leq T\left(r, f^{(k)}\right)-T(r, f)+N\left(r, \frac{1}{f}\right)+S(r, f) .
$$

Proof. By the first fundamental theorem and the lemma of logarithmic derivatives, we get:

$$
m\left(r, \frac{f^{(k)}}{f}\right)=S(r, f)
$$

so we can deduce

$$
\begin{aligned}
N\left(r, \frac{1}{f(k)}\right) & =T\left(r, f^{(k)}\right)-m\left(r, \frac{1}{f^{(k)}}\right)+O(1) \\
& \leq T\left(r, f^{(k)}\right)-\left(m\left(r, \frac{1}{f}\right)-S(r, f)\right)+O(1) \\
& \leq T\left(r, f^{(k)}\right)-\left(T(r, f)-N\left(r, \frac{1}{f}\right)+O(1)\right)+S(r, f) \\
& \leq T\left(r, f^{(k)}\right)-T(r, f)+N\left(r, \frac{1}{f}\right)+S(r, f) .
\end{aligned}
$$

This proves Lemma 3. 


\section{Proof of Theorem 1}

Proof of Theorem 1. We assume $f \not \equiv f^{(k)}$. Set:

$$
\frac{f^{(k)}-a}{f-a}=h \text {. }
$$

We distinguish the following two cases.

1: $h \equiv c(c \neq 1)$. From (3.1) we have:

$$
\frac{f^{(k)}}{a}-\frac{c f}{a}=(1-c),
$$

and hence by Lemmas 1 and 3 we have:

$$
\begin{aligned}
T\left(r, f^{(k)}\right) & \leq T\left(r, \frac{f^{(k)}}{a}\right)+S(r, f) \\
& \leq \bar{N}\left(r, \frac{a}{f^{(k)}}\right)+\bar{N}\left(r, \frac{a}{f}\right)+\bar{N}\left(r, \frac{f^{(k)}}{a}\right)+S(r, f) \\
& \leq T\left(r, f^{(k)}\right)-T(r, f)+2 N\left(r, \frac{1}{f}\right)+\bar{N}(r, f)+S(r, f),
\end{aligned}
$$

thus

$$
T(r, f) \leq 2 N\left(r, \frac{1}{f}\right)+\bar{N}(r, f)+S(r, f),
$$

from which we get

$$
2 \delta(0, f)+\Theta(\infty, f) \leq 2
$$

This contradicts (1.1)

2: $h \not \equiv$ const. From (3.1) we have;

$$
\frac{f^{(k)}}{a}-\frac{h f}{a}+h=1 .
$$

Set $f_{1}=f^{(k)} / a, f_{2}=-h f / a, f_{3}=h$, then

$$
\sum_{i=1}^{3} f_{i} \equiv 1 .
$$

We distinguish the following two subcases again.

CASE 2.1. $f_{1}, f_{2}, f_{3}$ are three linearly independent meromorphic functions, then by lemma 2 we have: 


$$
\begin{aligned}
T\left(r, f^{(k)}\right) \leq & T\left(r, \frac{f^{(k)}}{a}\right)+S(r, f) \\
\leq & N\left(r, \frac{a}{f^{(k)}}\right)+N\left(r, \frac{a}{f h}\right)+N\left(r, \frac{1}{h}\right)+N(r, D) \\
& -N\left(r, \frac{h f}{a}\right)-N(r, h)+o(T(r)) .
\end{aligned}
$$

as $r \rightarrow+\infty, r \in I$. On the other hand, since

$$
N(r, D)=N\left(r,\left(\frac{h f}{a}\right)^{\prime \prime} h^{\prime}-h^{\prime \prime}\left(\frac{h f}{a}\right)^{\prime}\right) \leq N\left(r,\left(\frac{h f}{a}\right)^{\prime \prime}\right)+N\left(r, h^{\prime \prime}\right),
$$

we have

$$
\begin{aligned}
N(r, D)-N\left(r, \frac{h f}{a}\right)-N(r, h) & \leq N\left(r,\left(\frac{h f}{a}\right)^{\prime \prime}\right)+N\left(r, h^{\prime \prime}\right)-N\left(r, \frac{h f}{a}\right)-N(r, h) \\
& \leq 2 \bar{N}\left(r, \frac{h f}{a}\right)+2 \bar{N}(r, h) .
\end{aligned}
$$

And since $f-a$ and $f^{(k)}-a$ share the value $0 \mathrm{CM}$ and $f^{(k)}$ and $a(z)$ do not have any common poles of same multiplicity, we know that $h \neq 0$. On the other hand, the pole of $h$ must be the pole of $f$ or $a(z)$, so

$$
\begin{aligned}
\bar{N}\left(r, \frac{h f}{a}\right) & \leq \bar{N}(r, f)+S(r, f) \\
\bar{N}(r, h) & \leq \bar{N}(r, f)+S(r, f) .
\end{aligned}
$$

From (3.3), (3.4), (3.5), we can get:

$$
\begin{aligned}
T\left(r, f^{(k)}\right) \leq & N\left(r, \frac{1}{f^{(k)}}\right)+N\left(r, \frac{1}{f}\right)+2 N\left(r, \frac{1}{h}\right) \\
& +2 \bar{N}(r, h)+2 \bar{N}(r, f)+S(r, f) .
\end{aligned}
$$

According to Lemma 3 and (3.6), we have

$$
T(r, f) \leq 2 N\left(r, \frac{1}{f}\right)+4 \bar{N}(r, f)+S(r, f),
$$

as $r \rightarrow+\infty, r \in I$, which contradicts (1.1).

CASE 2.2. $f_{1}, f_{2}, f_{3}$ are three linearly dependent meromorphic functions, namely there exist three constants which are not all equal zero and satisfy that:

$$
c_{1} f_{1}+c_{2} f_{2}+c_{3} f_{3}=0 .
$$

Obviously, $c_{1} \neq 0$. In fact, if $c_{1}=0$, then $c_{2} \neq 0, c_{3} \neq 0$, and by (3.7) we can obtain $h\left(c_{2}(f / a)-c_{3}\right)=0$, since $h \not \equiv$ const, $f=a c_{3} / c_{2}$. From this we can deduce: 
which is impossible.

$$
T(r, f)=T\left(r, \frac{a c_{3}}{c_{2}}\right)=S(r, f),
$$

By (3.2) and (3.7), we have

$$
\left(c_{2}-c_{1}\right) \frac{h f}{a}+\left(c_{1}-c_{3}\right) h=c_{1},
$$

We consider three subcases again.

2.2.1: $\quad\left(c_{2}-c_{1}\right) \neq 0,\left(c_{1}-c_{3}\right) \neq 0$, we obtain from (3.8)

and by Lemma 1

$$
\frac{\left(c_{1}-c_{2}\right)}{c_{1}} \frac{f}{a}+\frac{1}{h}=\frac{c_{1}-c_{3}}{c_{1}},
$$

$$
\begin{aligned}
T(r, f) & \leq T\left(r, \frac{f}{a}\right)+S(r, f) \\
& \leq \bar{N}\left(r, \frac{a}{f}\right)+\bar{N}(r, h)+\bar{N}\left(r, \frac{f}{a}\right)+S(r, f) \\
& \leq N\left(r, \frac{1}{f}\right)+2 \bar{N}(r, f)+S(r, f) .
\end{aligned}
$$

Similarly, this is impossible because of the condition (1.1).

2.2.2: $\left(c_{2}-c_{1}\right)=0,\left(c_{1}-c_{3}\right) \neq 0$, we deduce from (3.8),

$$
h=\frac{c_{1}}{c_{1}-c_{3}},
$$

which is a contradiction because of $h \not \equiv$ const.

2.2.3: $\left(c_{2}-c_{1}\right) \neq 0,\left(c_{1}-c_{3}\right)=0$, from (3.8) we can obtain

by (3.2) and (3.9), we get

$$
f h=\frac{a c_{1}}{c_{2}-c_{1}},
$$

$$
\frac{f^{(k)}}{a}+h=\frac{c_{2}}{c_{2}-c_{1}},
$$

If $c_{2} \neq 0$, then by Lemmas 1 and 3

$$
\begin{aligned}
T\left(r, f^{(k)}\right) & \leq T\left(r, \frac{f^{(k)}}{a}\right)+S(r, f) \\
& \leq \bar{N}\left(r, \frac{a}{f^{(k)}}\right)+\bar{N}\left(r, \frac{1}{h}\right)+\bar{N}\left(r, \frac{f^{(k)}}{a}\right)+S(r, f) \\
& \leq T\left(r, f^{(k)}\right)-T(r, f)+N\left(r, \frac{1}{f}\right)+\bar{N}(r, f)+S(r, f) .
\end{aligned}
$$


Therefore

$$
T(r, f) \leq N\left(r, \frac{1}{f}\right)+\bar{N}(r, f)+S(r, f),
$$

and similarly, this is impossible because of the condition (1.1).

If $c_{2}=0$, then we have

$$
f^{(k)}+a h=0 \Rightarrow f h=-a,
$$

so we can deduce from (3.11)

$$
f f^{(k)}=a^{2}
$$

We note that

$$
N\left(r, \frac{1}{f}\right) \leq N\left(r, \frac{1}{f f^{(k)}}\right)
$$

and

$$
\begin{aligned}
2 m\left(r, \frac{1}{f}\right) & =m\left(r, \frac{1}{f^{2}}\right) \\
& \leq m\left(r, \frac{f f^{(k)}}{f^{2}}\right)+m\left(r, \frac{1}{f f^{(k)}}\right) \\
& =S(r, f)+m\left(r, \frac{1}{f f^{(k)}}\right),
\end{aligned}
$$

so that

$$
T(r, f) \leq T\left(r, f f^{(k)}\right)+S(r, f) .
$$

Therefore we can get from (3.12) and (3.13)

$$
T(r, f) \leq T\left(r, f f^{(k)}\right)+S(r, f)=T\left(r, a^{2}\right)+S(r, f)=S(r, f),
$$

which is impossible.

This proves Theorem 1.

\section{Acknowledgment}

The authors want to thank the referee for his/her thorough review and valuable suggestions towards improved of the paper.

\section{REFERENCES}

[1] L. YANG, Value Distribution Theory, Springer-Verlag, Berlin, (1993).

[2] Chung-Chun Yang and Yi Hong-Xun, Uniqueness Theory of Meromorphic Functions, Kluwer Academic Publishers, (2004). 
[ 3 ] R. BRÜCK, On entire functions which share one value $\mathrm{CM}$ with their first derivatives, Result. in Math, 30 (1996), 21-24.

[ 4 ] G. G. Gundersen and L. Z. Yang, Entire Functions that share one value with one or two of their derivatives, J. Math. Anal. Appl., 223 (1998), 245-260.

[5] Kit-wing YU, On entire and meromorphic functions that share small functions with their derivatives, J. Inequal. Pure and Appl. Math, 2003, 4(1).

Department of Mathematics

Chongqing University, ChongQIng, 400044

CHINA 